\title{
Intradermal tranexamic acid in the treatment of melasma: A prospective study of 20 Moroccan cases
}

\author{
Meriem EI Mansouri, Fouzia Hali, Soumaya Chiheb
}

Department of Dermatology and Venereology, HUC Ibn Rochd, University Hassan II of Casablanca, Morocco

Corresponding author: Meriem El Mansouri, MD, E-mail: meriemelmans@gmail.com

\begin{abstract}
Background: The objective of this study was to evaluate the effectiveness and safety of tranexamic acid mesotherapy in the treatment of melasma in the Moroccan population. Materials and Methods: All the patients received an intradermal injection of tranexamic acid $(5 \mathrm{mg} / \mathrm{mL})$. No other local or general treatment was administered. The patients were kept under external photoprotection only. Pre- and post-treatment photographs were taken and analyzed with the Visioface ${ }^{\circledR}$ RD hardware. The evaluation was employed the MASI score. Results: All patients had a dark skin phototype. The average duration of melasma evolution was 3.67 years. The pre-treatment average MASI was 7.6. The post-therapeutic average MASI was 6 . The Visioface ${ }^{\circledR}$ RD hardware estimated a $25 \%$ reduction in the surface area attained at month five. No serious side effects were reported. Conclusion: Tranexamic acid was proven to be an essential tool in treating melasma.
\end{abstract}

Key words: Melasma; Tranexamic acid; Intradermal injection; Therapy

\section{INTRODUCTION}

Melasma is a chronic acquired hyperpigmentation of the skin characterized by irregular brown macules symmetrically distributed on sun-exposed areas of the body, especially the face.

It is a common cause of concern in dermatological care in the Moroccan population, affecting mainly females [1]. It has long been a condition annoying for both the physician and the patient.

So far, there is no therapy with fully satisfactory results. New therapeutic options have emerged, and some of these therapies have come under increasing focus. Tranexamic acid (TA, trans-4-(Aminomethyl) cyclohexane carboxylic acid (T-AMCHA)) is a plasmin inhibitor that has recently emerged as a promising new treatment for melasma, for which different galenic forms and ways of administration are proposed [2].

The primary objective of this study was to evaluate the effectiveness and safety of tranexamic acid mesotherapy in the treatment of melasma in the Moroccan population.

\section{MATERIALS AND METHODS}

A monocentric prospective study was conducted from December 2018 through May 2019 at the dermatology department of the Ibn Rochd University Hospital in Casablanca. The study included melasma patients over the age of eighteen years who had not received any treatment in the last two months prior to the study.

The study excluded pregnant and breastfeeding women and patients with a history of hypersensitivity to the product or other associated dermatosis, endocrine disorders, photodynamic therapy, a history of vitiligo, known abnormalities in wound healing, facial warts, herpes, and patients under anticoagulant treatment, on estrogen-progestin contraception, or with a known thromboembolic or cardiovascular disorder.

The injections were performed by a dermatologist in accordance with the following protocol: the skin was

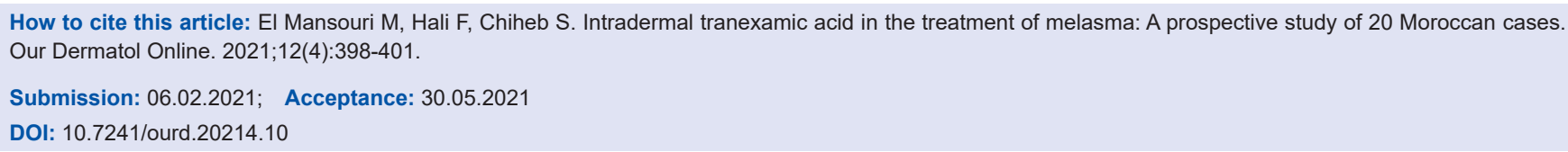


cleansed with saline solution, then an EMLA anesthetic cream was applied for thirty minutes.

All the patients received intradermal micro-injections of TA $(5 \mathrm{mg} / \mathrm{mL})$ every fifteen days for two months, then three cycles of the TA treatment repeated monthly.

Tranexamic acid by intradermal injection $(10 \mathrm{mg} / \mathrm{mL})$. Intradermal microinjections were performed by an insulin syringe with a 30-gauge needle. An interval of $10 \mathrm{~mm}$ between two injections was maintained. All aseptic conditions were respected. By the end of the procedure, cold compresses were applied followed by an SPF $50+$ cream.

The treatment was associated with currently external photoprotection by broad-spectrum sunscreen and $\mathrm{SPF} 50+$. No other local or general treatment was administered. The patients were kept under external photoprotection only.

Each patient gave their clear consent prior to the commencement of the study and was informed of the possible side effects of tranexamic acid.

Pre- and post-treatment photographs were taken and analyzed with the Visioface ${ }^{\circledR}$ RD hardware. This device was used to analyze the number of spots and the surface area affected. The evaluation employed the MASI score.

Improvements were judged as very good (disappearance of more than $75 \%$ of the lesions), good (disappearance of $50 \%$ to $75 \%$ of the lesions), moderate (disappearance of $25 \%$ to $50 \%$ of the lesions), incremental (disappearance of less than $25 \%$ of the lesions), or no response.

The tolerance assessment took into consideration the evaluation of pain with a simple verbal scale and any adverse reactions reported by patients or observed on check-ups.

The data collection form included:

- demographic characteristics (age, sex);

- pathological history and medication in use;

- risk factors (sun exposure, hormonal imbalance, the application of irritating topical products);

- the duration of lesions evolution;

- previous treatments (depigmentation, lasers, peelings).

The statistical analysis was performed with the SPSS software.

\section{Ethics Statement}

The study was approved by the institutional ethics committee of our institution, and all patients provided written informed consent.

\section{RESULTS}

Twenty patients were enrolled, all of which were females with an average age of 41.75 years (extremes: 2-65; standard deviation: 10.63).

Ten of the patients had a family history of melasma with sun exposure among all our patients. Eleven patients were previously pregnant. Ten of them were under long-term estrogen-progestin therapy. Previous treatments were used by nineteen patients: natural masks by eight patients, depigmenting agents without hydroquinone by eleven, hydroquinone by two, and peeling by three. Fifteen had already used several therapeutic combinations before the consultation. Three patients had facial hypersensitivity.

The average duration of melasma evolution was 3.67 years.

All patients had a dark skin phototype: six patients with phototype III, thirteen patients with phototype IV, and one patient with phototype $\mathrm{V}$. The clinical aspect was about pigmented macules, varying from light brown to dark brown.

The location of the melasma was centrofacial in eleven patients, malar in seven, and maxillary in two. The pretreatment average MASI was 7.6.

Interventional pain was considered moderate by five patients, intense by twelve, and unbearable by three. Post-interventional pain disappeared after one hour for all patients. Post-interventional erythema was reported by all patients and lasted for one day at most. No short-, medium-, or long-term side effects were recorded (eczematization, postinflammatory hyperpigmentation).

The patients were seen at Ml, M3, and M6. One patient reported a remarkably good improvement. A good improvement was noted by six patients. A moderate improvement was noted by four patients. Three patients reported a slight improvement. No response was noted by three patients. Three patients withdrew from the study. The post-treatment average 
Table 1: Protocols of intradermal TA reported by several studies

\begin{tabular}{lclcll}
\hline Study & No. of patients & Rhythm & $\begin{array}{c}\text { Duration } \\
\text { (in weeks) }\end{array}$ & Comparative treatment & Results \\
\hline Lee \& al. [11] & 100 & Weekly & 12 & None & Decrease in MASI. \\
Elfar \& El-Maghraby. [10] & 60 & Weekly & N/A & Silymarin, glycolic acid & Decrease in MASI. \\
Saki \& al. [12] & 37 & Monthly & 20 & Topical hydroquinone & No significant difference between the two groups. \\
Pazyar \& al. [13] & 49 & Fortnightly & 24 & Hydroquinone & No significant difference between the two groups. \\
\hline
\end{tabular}

MASI was 6. The Visioface® RD hardware estimated a $25 \%$ reduction in the surface area achieved at month five (Fig. 1).

\section{DISCUSSION}

Intradermal injection of tranexamic acid produced an improvement in melasma in our patients. No serious side effects apart from a mild local burning sensation and erythema were reported, and these were mostly transient.

Despite several proposed therapeutic modalities of variable efficiency and tolerance, melasma remains a therapeutic challenge that evolves chronically and recurrently [3]. Some topical agents, such as hydroquinone, are limited by complications, including irritant dermatitis, allergic contact dermatitis, postinflammatory hyperpigmentation, and exogenous ochronosis [4]. Darker skin types (Fitzpatrick types IV to VI) are particularly at risk for post-inflammatory hyperpigmentation (PIH) [5], which limits the use of topical bleaching formulas [6] and physical treatments as chemical peels and lasers in our context and forces us to evaluate the efficiency and safety of other modalities [4].

Tranexamic acid is a well-known hemostatic agent. In recent years, despite its mechanism of action remaining unclear, TA has been shown to produce encouraging results in the treatment of melisma [7]. Tranexamic acid inhibits the binding of plasminogen to keratinocytes, thereby reducing the synthesis of prostaglandins, which are known stimulators of tyrosinase activity. In addition, plasmin increases diffusible forms of vascular endothelial growth factor (VEGF), leading to angiogenesis. The inhibition of plasmin by TA leads to a reduction in angiogenesis, which allows it to act on the vascular component of melasma $[8,9]$.

Encouraging results were observed with different routes of TA administration (oral, topical, or microinjections), with a significant drop in the MASI score in all cases [9].

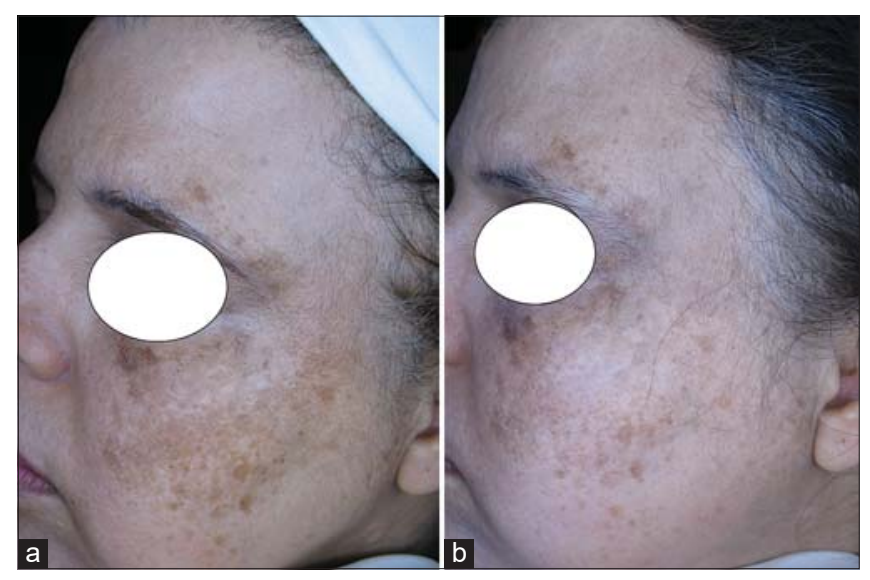

Figure 1: Photos (a) before and (b) after seven sessions of intradermal tranexamic acid therapy.

Nevertheless, given the potential for serious side effects with the use of oral TA, there has been an interest in evaluating TA as mesotherapy or topical therapy for melisma [10].

No definitive consensus on the use of TA for melasma currently exists. Several protocols have been proposed, with different concentrations and session rhythms. Variable results have been reported by trials of intradermal TA (Table 1).

TA has proven to be an essential tool in treating melasma [9].

The study was limited by a small number of patients, no control arm, and a short duration of the treatment and follow-up.

\section{CONCLUSION}

The encouraging results in our study as well as in the various studies reported make it possible to consider microinjection of TA as an effective option for the treatment of melasma in the Moroccan population. However, this data needs to be supported by larger randomized clinical trials to determine the most efficient mode of delivery. 


\section{Statement of Human and Animal Rights}

All the procedures followed were in accordance with the ethical standards of the responsible committee on human experimentation (institutional and national) and with the 2008 revision of the Declaration of Helsinki of 1975.

\section{Statement of Informed Consent}

Informed consent for participation in this study was obtained from all patients.

\section{REFERENCES}

1. Kettani F, Baline K, Hali F, Chiheb S. Melasma and tranexamic acid oral: prospective study of 15 cases. Ann Dermatol Venereol. 2019;146:A357-8.

2. Na JI, Choi SY, Yang SH, Choi HR, Kang HY, Park KC. Effect of tranexamic acid on melasma: A clinical trial with histological evaluation: Effect of tranexamic acid on melasma. J Eur Acad Dermatol Venereol. 2013;27:1035-9.

3. Pazyar N, Yaghoobi R, Zeynalie M, Vala S. Comparison of the efficacy of intradermal injected tranexamic acid vs hydroquinone cream in the treatment of melasma. Clin Cosmet Investig Dermatol. 2019;12:115-22.

4. Sheth VM, Pandya AG. Melasma: A comprehensive update. J Am Acad Dermatol. 2011;65:699-714.

5. Davis EC, Callender VD. Postinflammatory hyperpigmentation. J Clin Aesthet Dermatol. 2010;3:20-31.

6. Pollock S, Taylor S, Oyerinde O, Nurmohamed S, Dlova N, Sarkar R.
The dark side of skin lightening: An international collaboration and review of a public health issue affecting dermatology. IJWD.2020

7. Wang JV, Jhawar N, Saedi N. Tranexamic acid for melasma: Evaluating the various formulations. J Clin Aesthet Dermatol. 2019;12:E73-4

8. Kim SJ, Park JY, Shibata T, Fujiwara R, Kang HY. Efficacy and possible mechanisms of topical tranexamic acid in melasma. Clin Exp Dermatol. 2016;41:480-5.

9. Dashore S, Mishra K. Tranexamic acid in melasma: Why and how? Indian J Drugs Dermatol. 2017;3:61-3.

10. Elfar NN. Efficacy of intradermal injection of tranexamic acid, topical silymarin and glycolic acid peeling in treatment of melasma: A comparative study. J Clin Exp Dermatol Res. 2015;06.

11. Lee JH, Park JG, Lim SH, Kim JY, Ahn KY, Kim MY. Localized intradermal microinjection of tranexamic acid for treatment of melasma in Asian patients: a preliminary clinical trial. Dermatol Surg. 2006;32:626-31.

12. Saki N, Darayesh M, Heiran A. Comparing the efficacy of topical hydroquinone $2 \%$ versus intradermal tranexamic acid microinjections in treating melasma: A split-face controlled trial. J Dermatolog Treat. 2018;29:405-10.

13. Pazyar N, Yaghoobi R, Zeynalie M, Vala S. Comparison of the efficacy of intradermal injected tranexamic acid vs hydroquinone cream in the treatment of melasma. Clin Cosmet Investig Dermatol. 2019;12:115-22.

Copyright by Meriem El Mansouri, et al. This is an open access article distributed under the terms of the Creative Commons Attribution License, which permits unrestricted use, distribution, and reproduction in any medium, provided the original author and source are credited.

Source of Support: Nil, Conflict of Interest: None declared. 